\title{
Ring-enhancing brain lesions: Listeria monocytogenes abscesses
}

\author{
Andrew C. Berry ${ }^{1}$ (D) Landai Nguyen ${ }^{1}$. Theresa Yarbrough $^{1} \cdot$ Faheem Iqbal $^{1}$. \\ William K. Green ${ }^{2}$
}

Received: 31 August 2015/Accepted: 14 September 2015/Published online: 22 October 2015

(C) SIMI 2015

An 82-year-old woman with a history of bilateral temporal arteritis and atrial fibrillation presented with short-term memory loss and feeling of being off-balance the previous 1 week. She could not remember her grandchildren's names and birthdays, which her family deemed unusual. Neurological evaluation a year prior to admission did not show dementia or any other alarming neurological symptoms. The patient had a head computed tomography (CT scan) without contrast 2 days prior to admission with neurology that was concerning, and she was subsequently sent to our emergency department (ED) (Fig. 1a). She had biopsy-proven bilateral temporal arteritis 3 months prior to admission, and had been on a prednisone taper since. The vital signs on admission included: $35.9{ }^{\circ} \mathrm{C}$ (96.7), 166/79 mmHg, 65 beats/min, 17 breaths/min, $96 \%$ oxygen saturation on room air, and body mass index of 24 . The physical examination was unremarkable except for a moon facies, facial erythema, irregular irregular heart rate and rhythm, and right eye blindness. The patient was oriented to self, not time, and could name and slowly follow commands. Cranial nerves 2-12 were grossly intact. Strength $5 / 5$ in all limbs, but the patient was slow with movements. Sensation was grossly intact. Repeat head CT with contrast was performed and revealed three ring-enhancing lesions of the biparietal-occipital lobes surrounded by vasogenic edema and effacement of the occipital horn of the left lateral ventricle (Fig. 1b). There was no evidence of

Andrew C. Berry

aberry5555@gmail.com

1 Department of Medicine, University of South Alabama, 2451 Fillingim St., Mobile, AL 36617-2238, USA

2 Division of Infectious Diseases, Department of Medicine, University of South Alabama, Mobile, AL, USA hemorrhage, midline shift, or acute infarct. The head CT scan without contrast prior to admission showed two ringenhancing lesions (Fig. 1a). RPR, toxoplasma antibody, and HIV were all negative. Blood cultures revealed 1 of 2 Gram-positive rods, later confirmed as Listeria monocytogenes (Fig. 1c). Culture of the brain lesion biopsy further confirmed L. monocytogenes brain abscesses (Fig. 1d). The patient was switched from empiric vancomycin, ceftriaxone, and metronidazole to ampicillin $2 \mathrm{~g}$ IV Q4H to be continued for 6-8 weeks.

In patients presenting to the ED with acute confusion, neurological decline or deficits, or any other change in mentation, thorough clinical examination and neurological imaging are vital for proper medical workup. There are many possible culprits of ring-enhancing lesions such as radiation necrosis, metastasis, abscess, glioblastoma multiforme, subacute infarct, contusion, or lymphoma. It is imperative to delineate the cause of the ring-enhancing lesion, as the treatment varies extensively among the aforementioned causes. Groups at risk for listerial infection include pregnant women, immunocompromised, elderly, or debilitated adults with underlying diseases. Ingestion of listeria-contaminated food is likely the source of infection in all human listerial infections. In our case a particular brand of ice cream consumed by the patient with a recent history of United States listeria outbreak remains the likely culprit. In the immunocompetent human, L. monocytogenes exposure usually results in self-limited, febrile diarrheal gastroenteritis lasting 1-3 days. In the immunocompromised, gastrointestinal invasion can lead to bacteremia, with a predilection for the CNS, where it can cause meningitis, meningoencephalitis, or rhombencephalitis [1]. Although L. monocytogenes is a well-known cause of meningitis and encephalitis, brain abscesses caused by this organism are highly uncommon and reported to occur in 
Fig. 1 a Computed tomography (CT) of the brain without contrast showing two ring-enhancing lesions of the biparietal-occipital lobes with no evidence of hemorrhage, infarct, or midline shift (arrows). b Computed tomography (CT) of the brain with contrast showing three ring-enhancing lesions of the biparietal-occipital lobes (arrows) surrounded by vasogenic edema and causing mass effect with effacement of the occipital horn of the left lateral ventricle. There was no evidence of hemorrhage, infarct, or midline shift. c Gram stain with $\times 100$ magnification of the initial blood culture on admission showing gram positive rods, later confirmed as L. monocytogenes. d Gram stain of the brain abscess sample with $\times 100$ magnification showing gram positive rods, later confirmed as L. monocytogenes
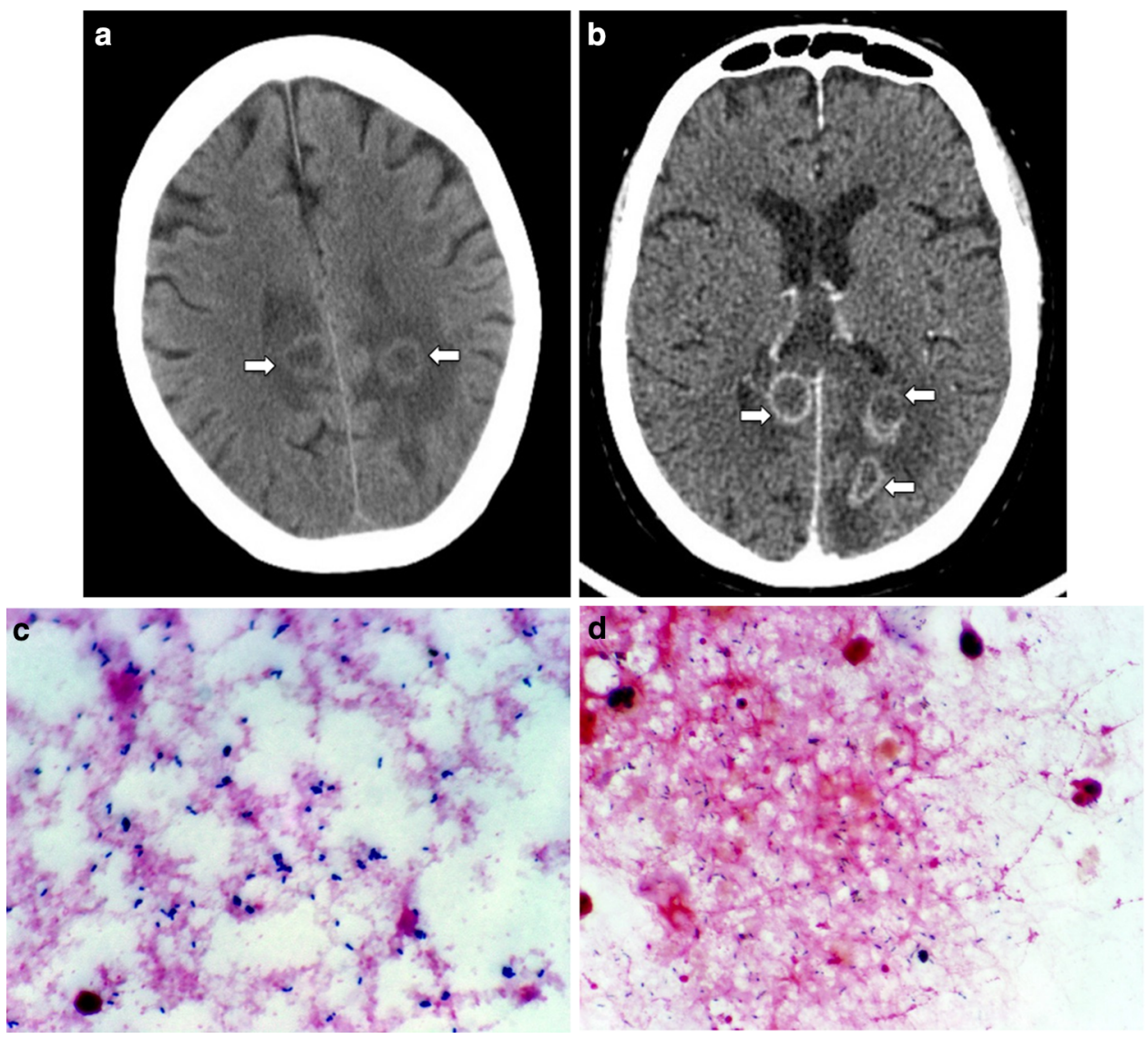

fewer than $10 \%$ of all listerial CNS infections [2]. Roughly 60 cases of listerial CNS brain abscesses have been reported to date, with over half attributed to an immunocompromised state [3].

As in our case, bacteremia is common, and present in about $86 \%$ of cases of listerial brain abscesses. This is a unique finding for listerial brain abscesses as bacteremia is only found in $11 \%$ of all other types of brain abscesses [4]. Mortality for listerial CNS abscesses has been reported to be $40 \%$, which is much higher than the $17 \%$ mortality for all other types of brain abscesses [5]. There remains no randomized controlled trial to establish a defined regimen for listeria brain abscess antimicrobial treatment. Current recommendations include IV ampicillin or trimethoprim-sulfamethoxazole if allergic to penicillins. Although adding IV gentamicin has been has been shown to have a synergistic effect in animal models, there remains no concrete evidence that it provides any additional benefit in humans [3]. In our case, we did not consider adding gentamicin because of its potential nephrotoxic adverse effects in an elderly women with already mild acute kidney injury. In addition, combination therapy of linezolid and rifampin for listeria brain abscesses has been considered in previous reports; however, there remains a paucity of evidence utilizing this regimen [3]. The only clear-cut recommendation remains IV antibiotic therapy for at least 6 weeks with follow-up serial neurological imaging until resolution of the brain abscesses.

\section{Compliance with ethical standards}

Conflict of interest The authors declare that they have no conflict of interest.

Statement of human and animal rights All procedures performed in studies involving human participants were in accordance with the ethical standards of the institutional/or national research committee and with the 1964 Helsinki Declaration and its later amendments or comparable ethical standards. This article does not contain any studies with animals performed by any of the authors.

Informed consent None.

\section{References}

1. Dalton CB, Austin CC, Sobel J et al (1997) An outbreak of gastroenteritis and fever due to Listeria monocytogenes in milk. N Engl J Med 336(2):100-105

2. Lorber B (2015) Listeria monocytogenes. In: Bennett J, Dolin R, Blaser M (eds) Mandell, Douglas, and Bennett's principles and practice of infectious diseases, 8th edn. Elsevier/Saunders, Philadelphia, pp 2383-2390 
3. Limmahakhun S, Chayakulkeeree M (2013) Listeria monocytogenes brain abscess: two cases and review of the literature. Southeast Asian J Trop Med Public Health 44(3):468-478

4. Mathisen GE, Johnson JP (1997) Brain absess. Clin Infect Dis 25:763-779
5. Cone LA, Leung MM, Byrd RG et al (2003) Multiple cerebral abscesses because of Listeria monocytogenes: three case reports and a literature review of supratentorial listerial brain abscess(es). Surg Neurol 59:320-328 